\title{
Research Article \\ Dual Feed, Single Element Antenna for WiMAX MIMO Application
}

\author{
Frank M. Caimi and Mark Mongomery \\ SkyCross, Inc. 7341 Office Park Place, Suite 102 Viera, FL 32940, USA \\ Correspondence should be addressed to Frank M. Caimi, frank.caimi@skycross.com
}

Received 11 December 2007; Accepted 10 April 2008

Recommended by Seong-Youp Suh

A novel u-shaped single element antenna having two feed ports is compared with two equal length monopoles separated by a distance equivalent to the width. A discussion of relative performance metrics is provided for MIMO applications, and measured data is given for comparison. Good impedance match and isolation of greater than $-10 \mathrm{~dB}$ are observed over the operating bandwidth from 2.3 to $2.39 \mathrm{GHz}$. The antenna patterns are highly uncorrelated, as illustrated by computation of the antenna pattern correlation coefficient for the two comparison monopoles.

Copyright (c) 2008 F. M. Caimi and M. Mongomery. This is an open access article distributed under the Creative Commons Attribution License, which permits unrestricted use, distribution, and reproduction in any medium, provided the original work is properly cited.

\section{INTRODUCTION}

With the continued proliferation of wireless devices comes the increasing need for data services for e-mail, world wide web access, video on demand, and streaming video. For those in the industry, this means providing reliable services without interruption, delay, and data corruption. Wireless carriers are forced to maintain an infrastructure to meet increasing needs for this reliability and higher data rates, creating the need for communications systems with higher capacity. Meeting these needs has been a challenge, but developments in technology continue to provide solutions or alternatives.

Multiple input, multiple output (MIMO) communications protocols are one such development along the path, and have demonstrated significant data communications capacity improvements by exploiting multiple independent spatio-temporal channels. These channels are found in signal environments where scattering and rotation of the electric vector of the signal are common - buildings, offices, homes, and so forth. Perhaps surprisingly, these techniques were used for military acoustic communication in the oceans prior to the coining of the term MIMO, since signals travel by discernibly different paths and often many paths are needed to ensure communications reliability. In order to exploit these channels, the antenna system should meet certain requirements. This paper reviews metrics used for characterization of antennas used in MIMO systems and covers advances in antenna technology that allow a single antenna to be used having multiple feed ports.

\section{ANTENNA METRICS FOR MIMO SYSTEMS}

In order to fully exploit independence of signal channels for the communications system, it is commonly believed that the transmitter and receiver must utilize multiple transducers (antennas) having certain characteristics. Since the signals may arrive from different directions, the antennas should exhibit characteristics that allow for highest independence of the received signals. One such characteristic is antenna pattern independence-measured by the pattern correlation coefficient [1] (although that alone is not sufficient, as it does not include the effect of the communications channel). The pattern correlation coefficient can be calculated from full sphere antenna pattern measurements and is given in terms of the electrical field components by [1]

$$
\rho_{p}=\frac{\int_{0}^{2 \pi} \int_{0}^{\pi} A_{12} d \Omega}{\sqrt{\int_{0}^{2 \pi} \int_{0}^{\pi} A_{11} d \Omega} \sqrt{\int_{0}^{2 \pi} \int_{0}^{\pi} A_{22} d \Omega}},
$$

where $A_{m n}(\theta, \varphi)=X E_{m v}(\theta, \varphi) E_{n v}^{*}(\theta, \varphi)+E_{m h}(\theta, \varphi) E_{n h}^{*}(\theta, \varphi)$, and the cross-polarization power ratio $X=\frac{S_{v}}{S_{h}}$. 
The subscript indices $(m, n)$ refer to either antenna port 1 or 2, depending on their integer value; $\Omega(\theta, \phi)$ is the spatial angle in steradian as commonly depicted; and $E_{1, v}, E_{1, h}, E_{2, v}$, and $E_{2, h}$ are the complex envelopes of either the vertical or horizontal field components resulting from excitation of either antenna port 1 or 2 , respectively. The cross polarization ratio is defined as the ratio of the mean received power in the vertical polarization to the mean received power in the horizontal polarization, as shown.

Correlation coefficients less than a prescribed threshold are desirable, and envelope correlations $\left(\rho^{2}\right)$ less than 0.5 are preferred for acceptable data capacity according to many studies.

Antennas should not only exhibit low port-to-port signal correlation, but should also exhibit isolation from one another so that signals being broadcast or received from one feed port do not appear at the opposite port or feed. The isolation is typically measured at the antenna terminals by using the commonly derived $s$-parameters. Isolation figures are typically given in decibels and are designated as $S_{12}$ or $S_{21}$ depending on whether the measurement is from antenna 1 to 2 or vice versa. If the isolation is poor, the source antenna will deliver substantial power into the adjacent antenna's termination impedance (typically 50-ohms resistive), with a corresponding reduction of the overall radiation efficiency. $S_{21}$ values of $-10 \mathrm{~dB}$ are regularly deemed acceptable and resulting in power loss of less than $10 \%$.

Since the antenna near-field coupling is related to the far-field pattern, it is possible to approximately express the correlation coefficient in terms of the $s$-parameters measured at each antenna terminal. This is given by [2]

$$
\left|\rho_{s}\right|=\frac{\left|S_{11}^{*} S_{12}+S_{21}^{*} S_{22}\right|}{\left(1-\left(\left|S_{11}\right|^{2}+\left|S_{21}\right|^{2}\right)^{1 / 2}\left(1-\left(\left|S_{22}\right|^{2}+\left|S_{12}\right|^{2}\right)^{1 / 2}\right.\right.} .
$$

Comparison of measurements taken by this approach can approximate those computed using the pattern method, and are much simpler. Most studies and specifications for correlation coefficient refer to the envelope correlation which is approximately equal to the magnitude squared of $\rho$ as given in (1) and (2). From this equation, however, it is clear that small values for $S_{12}$ and $S_{21}$ can result in small values for $\rho_{s}$. There are, however, cases where the correlation coefficient might be small even if $S_{12}$ and $S_{21}$ are larger, depending on the input match reflected in the $S_{11}$ and $S_{22}$ values.

In spite of the ease by which the correlation coefficient can be computed using the previous equations, prediction of the communications system performance also relies on some measure of the signal independence resulting from the environment. This factor has been investigated and can be included in the antenna pattern correlation coefficient calculation to produce a more complete measure of the received signal correlation or independence in nonlaboratory conditions. The method involves the use of a simple environmental model that has been experimentally verified [3]. In this model, the signal power is described as a function of angle of arrival by probability functions $P_{v}$ and $P_{h}$, which can be included in (1) as follows:

$$
\begin{aligned}
A_{m n}(\theta, \varphi)= & X E_{m v}(\theta, \varphi) E_{n v}^{*}(\theta, \varphi) P_{v}(\theta, \varphi) \\
& +E_{m h}(\theta, \varphi) E_{n h}^{*}(\theta, \varphi) P_{h}(\theta, \varphi) .
\end{aligned}
$$

Measurement data have been taken to define parameters for Gaussian forms of $P_{v}$ and $P_{h}$ in various environments [4]. This data supports the general methodology for computing $\rho$ based on a field model (versus that of pattern alone) with good correlation between the two computational approaches. Perhaps most interesting is that the correlation coefficient depends primarily on the phase difference between the two antenna patterns-indicating that good correlation performance requires careful consideration of the antenna placement and design in the case, where multiple single antennas are used in close proximity.

Briefly then, the antenna system, its associated pattern, and the environment are all clearly important in establishing independence between the signals received at each antenna, and therefore the overall system data transfer rate.

\section{MULTIPLE VERSUS SINGLE ANTENNA DESIGN APPROACHES}

A standard approach to achieve MIMO operation is to develop multiple antennas that are sufficiently separated to achieve a desired level of signal independence and port-toport isolation. In small handheld terminals, this approach taxes the industrial design process to accommodate double or triple the space allocation commonly reserved for the antenna, as well as requiring additional feedline length. Perhaps the most stressing geometric configurations are for USB form factors requiring HSPA or WiMAX operational capability, since space allocation is extremely smallnominally $20 \mathrm{~mm}$ wide by $10 \mathrm{~mm}$ long.

Conversely, if it were possible to develop a single antenna having multiple feed ports, with equivalent or better performance characteristics than the separate antenna approach; it would represent a significant advantage by reducing the cost of assembly and manufacture while providing equivalent performance in an equal or perhaps smaller volume. Several studies have been reported in the literature describing potential antenna designs for MIMO applications in small handheld terminals. Generally, these make use of external lumped element decoupling networks between the feed ports to allow matching of even and odd modes to a common impedance, thereby producing small cross correlation and maximum gain over a limited frequency range [5]. In one recent publication, a ground wall and connecting line were used in conjunction with shorting pins to develop a high isolation two port MIMO antenna consisting of 2 folded monopoles for WiBro service in Korea [6]. Here, we report on a single antenna with 2 feeds and two unique resonance modes that occur at the same frequency. Operation of the antenna can be described by an electromagnetic model that illustrates the self-induced and conducted currents in relation to an active reference feedpoint. The opposite, symmetrically located feedpoint of the antenna is isolated from the reference feedpoint by $10 \mathrm{~dB}$ or more versus that 


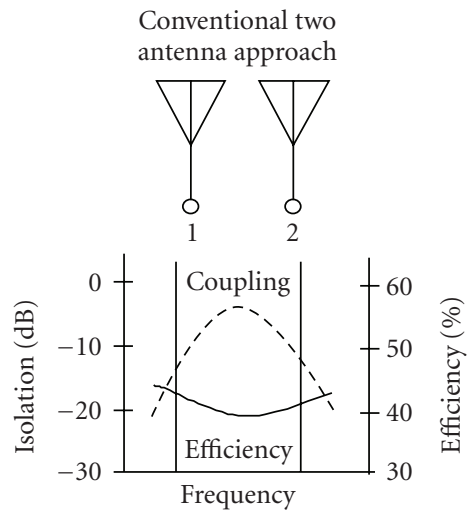

(a)

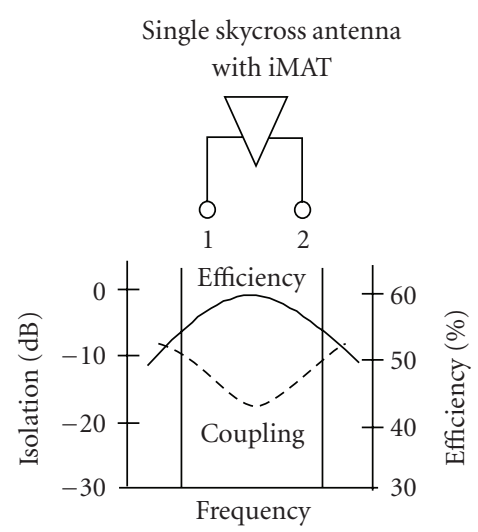

(b)

FIGURE 1: Illustration of a conventional antenna versus a two port iMAT antenna for MIMO application.

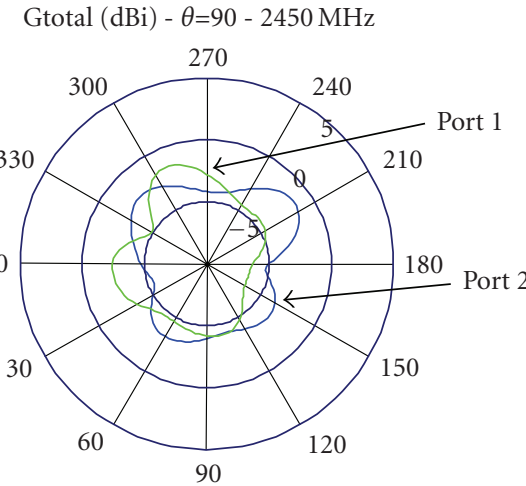

- Antenna 1

- Antenna 2

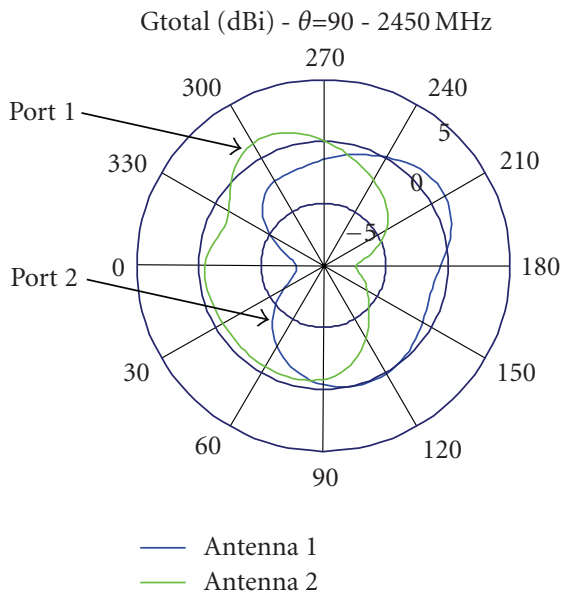

(b)

FIGURE 2: Example of antenna azimuthal gain pattern produced by: (a) two closely spaced monopoles and (b) single element iMAT antenna of similar size showing substantially different far-field pattern compared to (a).

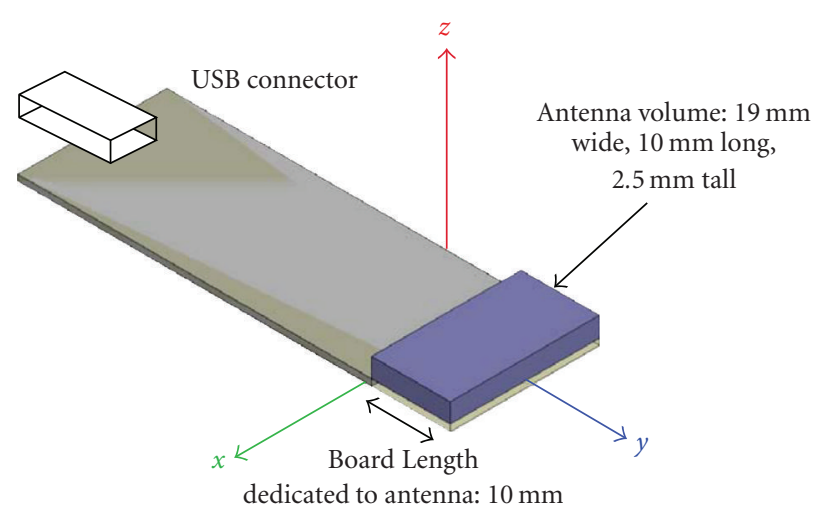

FIgUre 3: Example of WiMAX USB antenna illustrating small antenna volume of $19 \mathrm{~mm}$ wide by $10 \mathrm{~mm}$ high and extending away from PCB distal end by $10 \mathrm{~mm}$, [7].

achievable from two separate single antennas occupying the same or larger physical volume, and polarized equally. This method will be referred to as an isolated mode antenna technology or "iMAT" in this paper. A novel feature of the method is that the isolation improves as the source is swept through the resonance frequency, as compared with 2 adjacent monopole antennas.

The basic advantages of the single element MIMO antenna using iMAT versus the use of multiple antennas are illustrated in Figure 1. The coupling for the conventional antennas increases in the band region of interest, while for the isolated mode antenna, it decreases, as shown. In the conventional paired antenna arrangement, the current in the antenna increases toward resonance, which due to coupling to the adjacent antenna results in decreased efficiency. In contrast to the conventional antenna case, the iMAT approach relies on the electromagnetic modal distribution to achieve isolation, and therefore the frequency at which the modes are supported results in improved isolation as well as high-radiation efficiency as resonance is approached.

In the following example, the antenna pattern for the iMAT antenna is also substantially different from the 


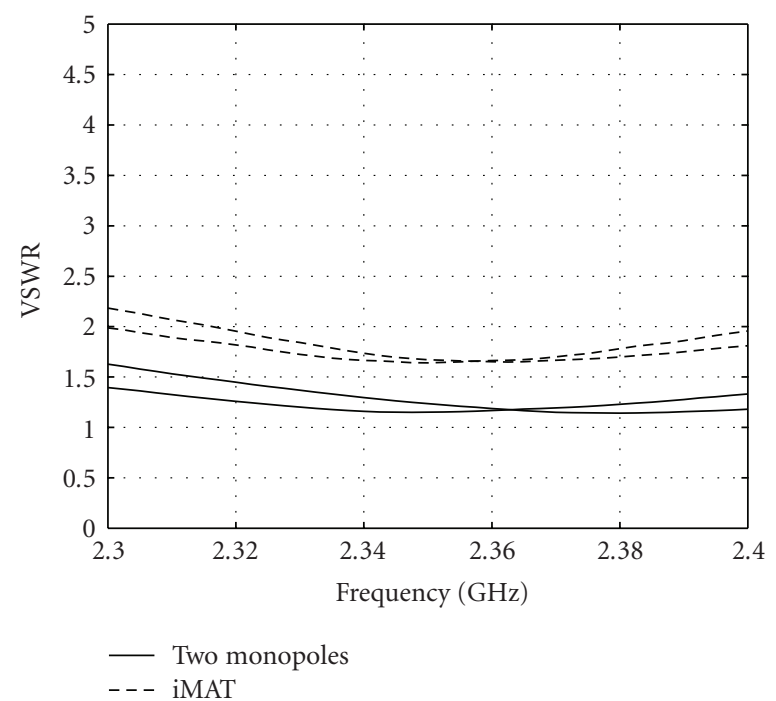

(a)

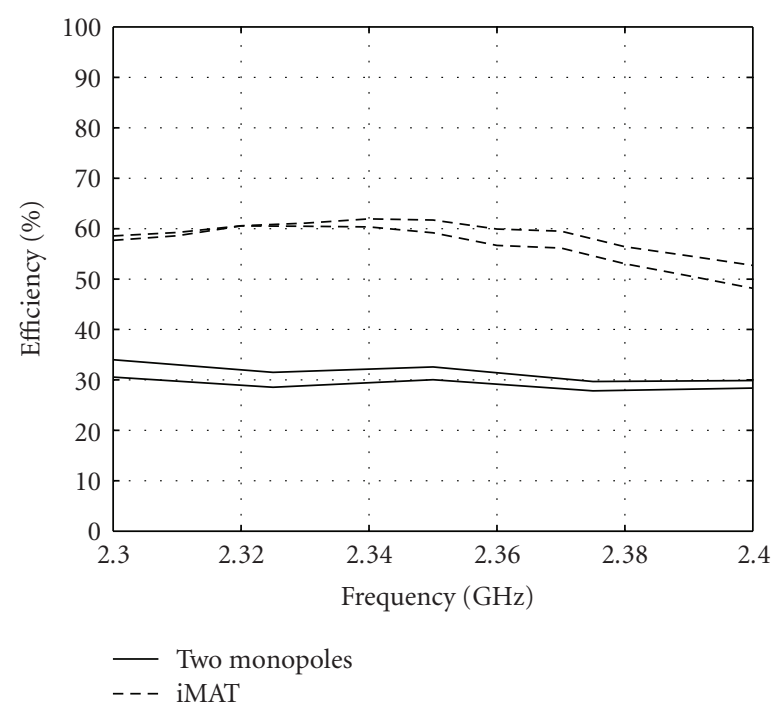

(c)

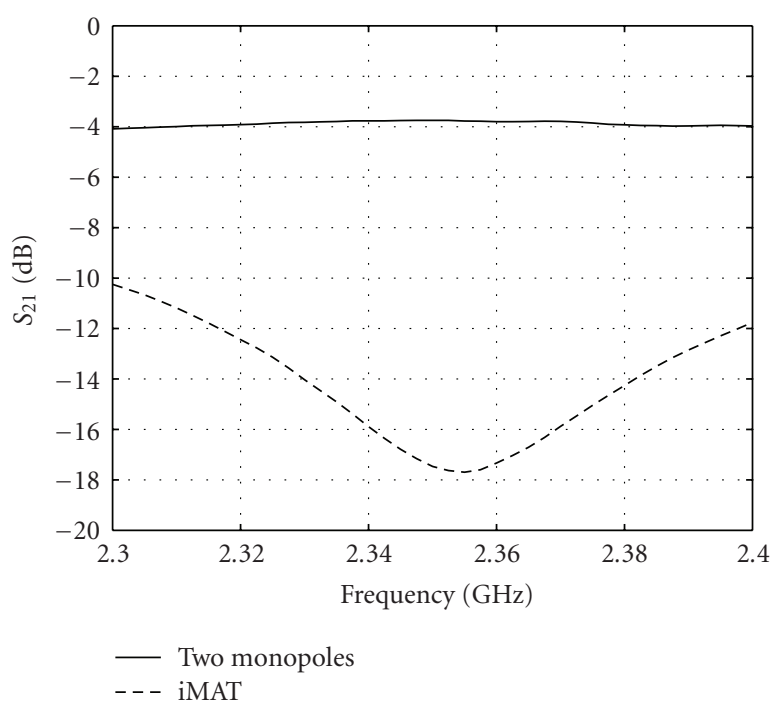

(b)

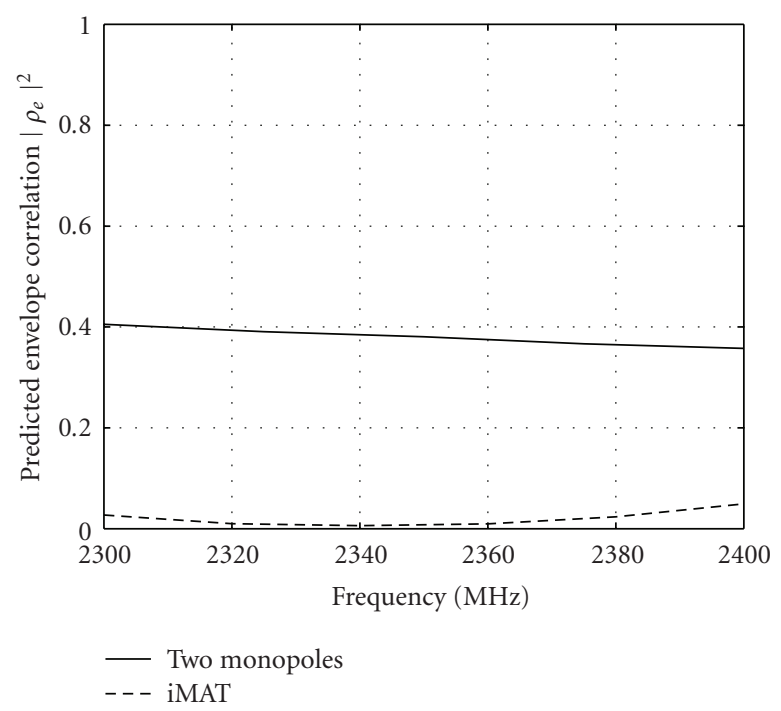

(d)

FIGURE 4: Comparison of (a) VSWR, (b) $S_{21}$, (c) radiation efficiency, and (d) envelope correlation coefficient for 2 cases: two monopoles and iMAT antenna over the frequency range from 2.3 to $2.4 \mathrm{GHz}$.

independent antenna case, as in Figure 2. The antenna pattern exhibits independence depending on which feed or port is excited, and therefore the correlation coefficient computed from the pattern is generally smaller than with independent separately located antennas in close proximity. An example of the pattern differences as shown in Figure 2. In Figure 2, the meanderline-loaded monopoles are separated by an average $10 \mathrm{~mm}$ or approximately $1 / 12$ wavelength in vacuum, and consequently are highly coupled. The antenna pattern is tilted away from azimuth due effects from the counterpoise, which accounts for the smaller gain, as compared to a free space dipole configuration. In addition, the coupling into the adjacent load resistance accounts for some additional gain reduction. The far-field pattern differences due to separate port excitation are generally expected to provide an improvement in signal independence by sampling different spatial channels.

The performance of an antenna using iMAT is best illustrated by example against other designs using the same space allocation. We have purposely chosen a monopole type of design, although it is possible to use a balanced antenna with similar results.

\section{IMAT ANTENNA DESIGN EXAMPLE}

An example of a USB WiMAX antenna design suited for the 2.3-2.4 GHz band is illustrated in Figure 3. A USB device typically consists of a printed circuit board (PCB) assembly, enclosed in a plastic housing, with USB connector at one end. In this case, the space available for the antennas is 
assumed to be at the opposite end of the PCB from the USB connector. The width of the device is $20 \mathrm{~mm}$ and the length and height available for the antennas are $10 \mathrm{~mm}$ and $2.5 \mathrm{~mm}$, respectively. The majority of the PCB, between the antennas and the USB connector, is taken to be a continuous RF ground.

\section{COMPARISON STUDY}

A similar antenna designed for the 2.3-2.4 GHz WiMAX/ WiBRO band is illustrated in Figure 4. Two different antenna solutions are taken for comparison. The first consists of two meanderline monopoles in the same form factor as illustrated in Figure 3. The distance from the ground available for the radiator is $10 \mathrm{~mm}$, or $\lambda / 12$ in free-space; however, the monopoles are made an effective $\lambda / 4$ length by using a meanderline conductor. The second case considers the iMAT solution that includes a similar meanderline structure for compactness, but one that maintains a half-wave equivalent electrical length as a single resonator. The iMAT solution in this case uses a half-wave mode of a u-shaped element, where the location of each feed port is symmetrically positioned and selected for the minimum coupling to the opposite feed port. A simulation analysis of the current developed by excitation at a single port shows that two fundamental competing modes contribute to a null condition at the opposing port, which is also supported by an analytic approach using transmission line theory of the u-shaped structure.

A comparison of measured performance for the two solutions is shown in Figure 4. Each approach yields good impedance match with a VSWR of 2 or better over the band (Figure $4(\mathrm{a}))$. However, the coupling $\left(S_{21}\right)$ for the monopoles is poor-about $-4 \mathrm{~dB}$ as shown in Figure $4(\mathrm{~b})$. In as similar application, the addition of the ground extension between the antennas provides marginal improvement of $S_{21}$ to about $-6 \mathrm{~dB}$ [7]. The iMAT solution has considerably better isolation, with $S_{21}$ values between -10 and $-15 \mathrm{~dB}$ in the designated band as shown in Figure 4(b).

The $S_{21}$ value has a direct impact on efficiency due to signal lost to the neighboring antenna and its associated load; and so the iMAT solution shows better efficiency than the other solution as shown in Figure 4(c). Similarly, the iMAT solution produces more diverse antenna patterns as indicated by the graph of correlation coefficients calculated using (1) as shown in Figure 4(d).

\section{SUMMARY}

Results have been presented for a novel antenna that uses a higher-order modal excitation from 2 separate feedpoints to achieve isolation of $10 \mathrm{~dB}$ or more over a limited bandwidth. The antenna size is similar to that of two identical monopole or dipole antennas that are closely coupled at less than 0.15 wavelength spacing. In addition, the antenna correlation coefficient for this approach is superior to the case of two separate closely spaced monopoles.

\section{REFERENCES}

[1] S. D. Parson, The Mobile Radio Propagation Channel, John Wiley \& Sons, New York, NY, USA, 2nd edition, 2000.

[2] S. Blanch, J. Romeu, and I. Corbella, "Exact representation of antenna system diversity performance from input parameter description," Electronics Letters, vol. 39, no. 9, pp. 705-707, 2003.

[3] G. Breit and E. Ozaki, "Phone level radiated test methodologies for multi-mode multi-band systems," in Proceedings of the International Wireless Industry Consortium Interactive Technical Workshop, Handset Antenna Technologies for MultiMode-MultiBand (IWPC '07), Durham, NC, USA, January-February 2007.

[4] K. Kalliola, K. Sulonen, H. Laitinen, O. Kivekas, J. Krogerus, and P. Vainikainen, "Angular power distribution and mean effective gain of mobile antenna in different propagation environments," IEEE Transactions on Vehicular Technology, vol. 51, no. 5, pp. 823-838, 2002.

[5] H. J. Chaloupka and X. Wang, "Novel approach for diversity and MIMO antennas at small mobile platforms," in Proceedings of the 15th IEEE International Symposium on Personal, Indoor and Mobile Radio Communications (PIMRC '04), vol. 1, pp. 637-642, Barcelona, Spain, September 2004.

[6] K. Chung and J. H. Yoon, "Integrated MIMO antenna with high isolation characteristic," Electronics Letters, vol. 43, no. 4, pp. 199-201, 2007.

[7] Skycross, Inc, iMAT Antenna Whitepaper, January 2008, http://www.skycross.com/. 

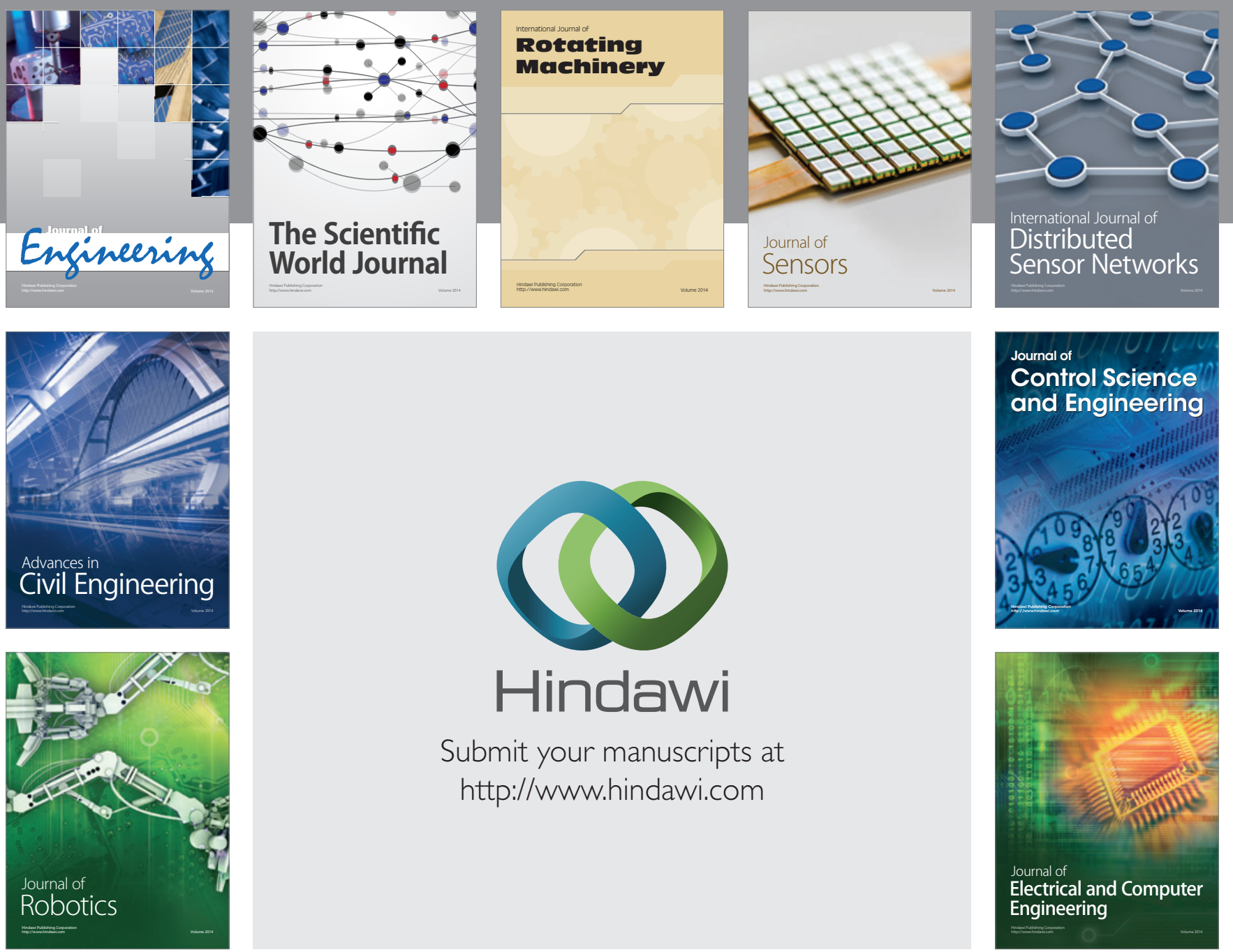

Submit your manuscripts at

http://www.hindawi.com
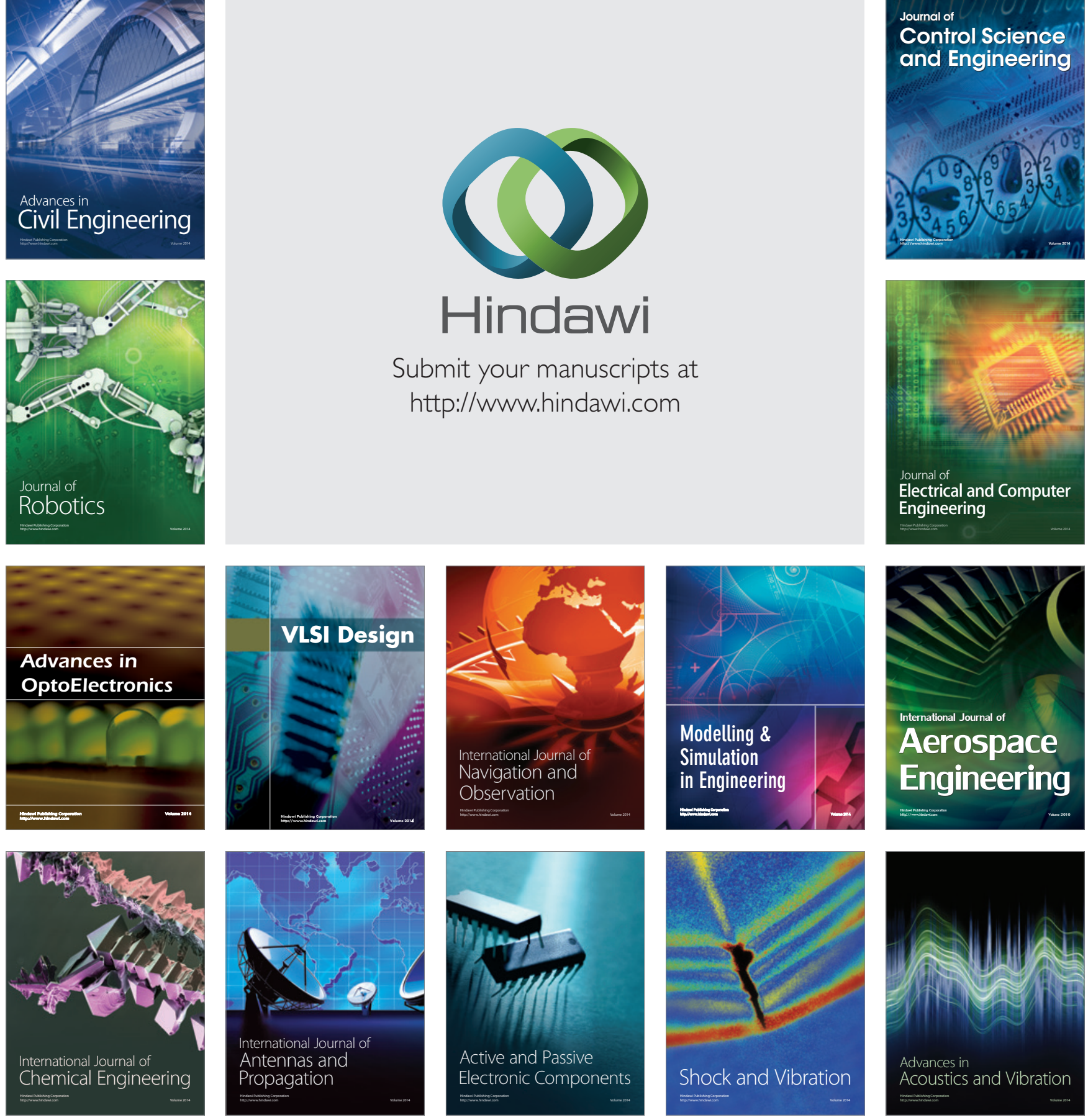